\title{
A SENSOR AIDED H.264/AVC VIDEO ENCODER FOR AERIAL VIDEO SEQUENCES WITH IN THE LOOP METADATA CORRECTION
}

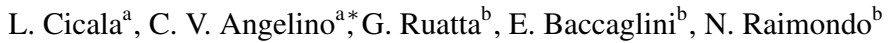 \\ ${ }^{a}$ CIRA, the Italian Aerospace Research Centre, 81043 Capua, Italy - (1.cicala, c.angelino)@ cira.it \\ ${ }^{\mathrm{b}}$ Istituto Superiore Mario Boella, Torino, Italy - (ruatta, baccaglini, raimondo)@ismb.it
}

Commission III, WG III/3

KEY WORDS: UAV, sensor aided video coding, metadata correction, h.264, x264, vision aided navigation, sensor fusion

\begin{abstract}
:
Unmanned Aerial Vehicles (UAVs) are often employed to collect high resolution images in order to perform image mosaicking and/or $3 \mathrm{D}$ reconstruction. Images are usually stored on board and then processed with on-ground desktop software. In such a way the computational load, and hence the power consumption, is moved on ground, leaving on board only the task of storing data. Such an approach is important in the case of small multi-rotorcraft UAVs because of their low endurance due to the short battery life. Images can be stored on board with either still image or video data compression. Still image system are preferred when low frame rates are involved, because video coding systems are based on motion estimation and compensation algorithms which fail when the motion vectors are significantly long and when the overlapping between subsequent frames is very small. In this scenario, UAVs attitude and position metadata from the Inertial Navigation System (INS) can be employed to estimate global motion parameters without video analysis. A low complexity image analysis can be still performed in order to refine the motion field estimated using only the metadata. In this work, we propose to use this refinement step in order to improve the position and attitude estimation produced by the navigation system in order to maximize the encoder performance. Experiments are performed on both simulated and real world video sequences.
\end{abstract}

\section{INTRODUCTION}

Nowadays, collection of data is the most important purpose of any Unmanned Aerial Vehicles (UAVs) (Chen et al., 2011). In military applications, long range UAVs can penetrate areas and locations that manned expeditions cannot effort without exceptional risks. Often this task is achieved using a set of on-board HD digital video cameras. The STANAG 4609 standard (NATO, 2009) is currently employed in NATO military UAV applications and adopts MPEG-2 and H.264/AVC standards for both Standard (SD) and High Definition (HD) motion imagery coding. However, these standards have been developed for general purpose video encoding and do not exploit the typical features of aerial video sequences. For instance, during the completion of mission, it is often required for UAVs to operate BLOS (Behind Line of Sight), a condition in which the direct communication between the vehicle and the ground control station is unfeasible. In this scenario the motion imagery acquired from payload sensors must be uploaded on a satellite data-link whose bandwidth is usually limited. It is then unlikely to achieve a high frame rate acquisition even because additional data gathered by the other payload sensors share the same data-link and further reduce the bandwidth available to the video stream. In civilian application miniUAVs, i.e., small remote piloted multirotorcrafts, are used in line of sight, often to acquire high resolution images for mosaicing and/or 3D reconstruction. During the mission, these high resolution data are stored on-board, while only the video coming from the first person view camera is sent on-ground to the remote pilot. In this situation the main mission constraint is the duration of the battery that supplies the vehicle.

In both the mentioned situations, it can be desirable to optimize the available resources (bandwidth, power supply) in order to improve the mission performance (more data, more flight time). When high frame rate videos are not a desiderata of the mission,

${ }^{*}$ Corresponding author one solution can be the reduction of the acquisition frame rate. In such a scenario, the video sequences are sent/stored at few frames per second (fps) and hence the overlap between two consecutive frames is lower than standard video streams. Usually at low frame rates the commercial video encorders fail in performing a good motion estimation/compensation, due to the length of the motion vectors and to the prospective changes among frames that make hard the motion vector prediction. In such situations a still image encoder can be more or equally performing.

However, the motion of a camera on an UAV is not unpredictable, but can be derived by the position and orientation data delivered by the on-board navigation systems. These data are often embedded in the transport bitstream (NATO, 2009), together with the encoded video, as "metadata", in the same way as the subtitles are multiplexed with the video in the commercial digital television. Besides the geometry of the overflight scene is roughly known and can be approximately estimated using, for example, a laser altimeter, or the GPS position and a Digital Terrain Model (DTM). With such information, a Global Motion (GM) in the image plane can be inferred without computationally intensive video analyses. In (Bhaskaranand and Gibson, 2015) authors investigate a low complexity encoder with GM based frame prediction and no block Motion Estimation (ME). For fly-over videos, it is shown that the encoder can achieve a $40 \%$ bit rate savings over a h.264 encoder with ME block size restricted to $8 \times 8$ and at lower complexity. In (Morimoto et al., 1997) and (Steinbach et al., 1999) GM parameters are used to compensate frames that are used as reference for block ME using GM within standard MPEG-4 and H.264 codecs. In (Gong et al., 2010) authors propose a framework tailored for UAV applications that uses the GM information and an homography model to code the stream using JPEG2000. In (Soares and Pinho, 2013) and (Angelino et al., 2013b) authors present modifications of the H.264/AVC encoder to initialize the motion vectors (MVs) using the camera motion information from UAV sensors. These latter approaches perform block ME at a lower complexity, and transmit the derived block 
MVs. Both approaches guarantee the generation of a standardcompliant H.264/AVC bitstream, thus no changes at the decoder side are required.

In this paper, as a natural continuations of the studies reported in (Angelino et al., 2012, Angelino et al., 2013b) and (Angelino et al., 2013a), we propose a sensor aided video encoder to be used at high resolution and low frame rates on aerial video sequences. The proposed encoder is obtained modifying the open source implementation of h.264/AVC video coding standard (ISO/IEC, 2006) x264 (Merritt and Rahul, 2006) and fully compliant with h.264. As opposed to the previous works, here the problems of video coding and of the needed metadata correction are tackled in the same integrated design. This paper is focused on the improvements in terms of rate-distortion performance. Moreover, further aspects about a sensor aided encoder design, unpublished results and considerations are reported.

The remainder of this paper is organized as follows. Section 2. introduces the proposed sensor aided coding scheme with in the loop metadata correction. Section 3. presents in details the modifications to the original $\mathrm{x} 264$ open source code and reports a comparison of the computational complexity of the proposed approach with the original scheme. Section 4. describes how to improve the navigation data using the optical flow calculated by the proposed video encoder.In Section 5. experimental results are presented and in Section 6. conclusions and future work are discussed.

\section{SENSOR AIDED VIDEO CODING}

\subsection{Encoding scheme}

The h.264 standard design follows the so-called block based hybrid video coding approach in which each coded picture is represented in block-shaped units. The approach is hybrid because inter-picture prediction is used to exploit temporal statistical dependencies and transform coding of the prediction residual is the performed to exploit spatial statistical dependencies. The blocks can be predicted in Intra (from blocks of another frame) or Inter mode (from blocks of the same frame). The Inter prediction can be of type Predicted (from a single frame), or of type Bipredicted (from two frames, one belonging to the previously acquired frames, the other belonging to the successively acquired frames). If type Bi-predicted frames are used, the encoder must wait some frames before to encode the current frame, introducing a delay. For every Group Of Pictures (GOP) to encode, the sequence of I (Intra), P (Predicted) and B (Bi-Predicted) frames is determined by a GOP decision algorithm.

The Inter prediction is performed by a ME step, in witch the translational motion vector is estimated for every block of the video frame to encode, followed by a motion compensation process, in witch the block of the previously encoded (and decoded) video frame is translated on the current block position. In (Angelino et al., 2013b) the authors propose to modify a well known open source implementation of a standard h.264 encoder, x264, which uses the metadata provided by the navigation system of the UAV in order to accurately predict the MVs, at low frame rates also. Experimental results showed that in such way both better quality $v s$ bitrate tradeoffs and lower computational complexity can be reached.

In this work we present a similar encoder, whose structure is shown in Figure 1. A common h.264 encoding scheme is modified in order to take in account of metadata (position and orientation) coming from the navigation system of the UAV. The camera is supposed to be internally and externally calibrated with respect

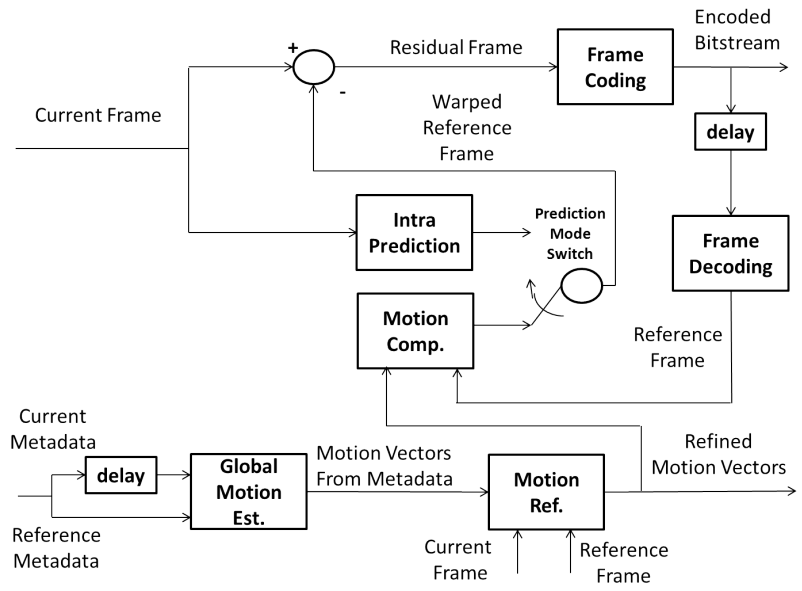

Figure 1: Sensor aided video encoder scheme.

to the navigation system. A Global Motion Estimation (GME) is performed using metadata and a rough planar representation of the overflight scene (i.e., supposing the ground an horizontal plane and using an altimeter to determine the distance of the aerial platform from the ground). A further MV refinement is performed by block matching, as proposed in the original version of x264, but starting from a more accurate initial estimate of the MVs, as provided by the GM estimation module.

Further, in addition to the scheme presented in the cited work, the proposed solution uses the estimated motion field as optical flow estimation for a state-of-the-art camera egomotion algorithm based on RANSAC homography model estimation and algebraic motion data extraction. The camera egomotion is used in loop with an Unscented Kalman Filter (UKF) in order to refine the position and orientation data provided by the navigation system. Such use of the motion field will be discussed in the Subsection 4.

Figures 2 and 3 show a situation in which the ME algorithm of x264 fails while the proposed ME process, initialized with the sensor based GME, performs with success. The vectors in overlay represent the motion vectors found by the ME process. When an appropriate MV cannot be estimated, Intra prediction is performed instead.

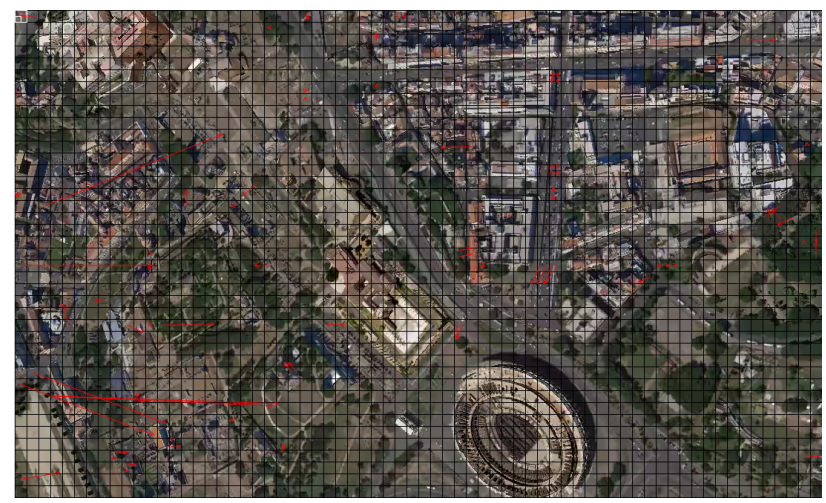

Figure 2: An example of failure of the motion estimation at low frame rates $(0.5 \mathrm{fps})$.

\subsection{Sensor aided Global Motion estimation}

In this paragraph it is showed how to obtain the homography transformation between two frames processing the synchronized 


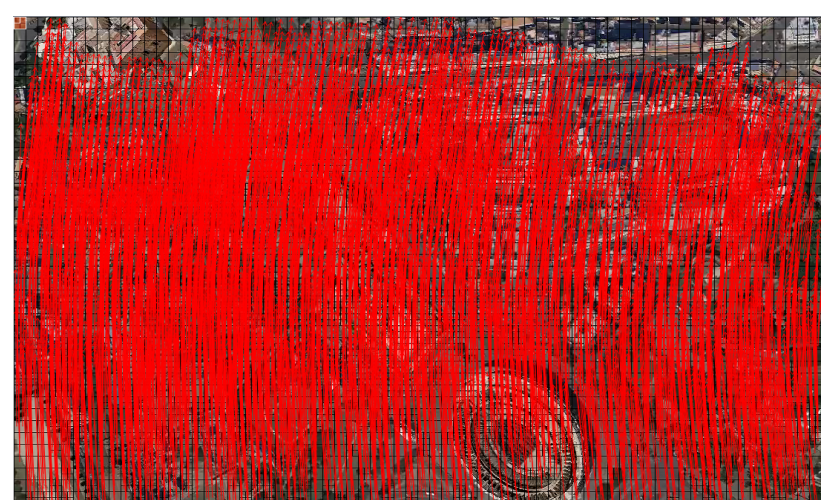

Figure 3: An example of correct motion estimation initialized by a sensor aided global motion estimation. The analyzed video is the same of Figure 2.

metadata in order to initialize the motion field (Global Motion estimation).

We first introduce some notation. Matrices are denoted by capital italics. Vectors are denoted by bold fonts either capital or small. A three-dimensional column vector is specified by $\left(s_{1}, s_{2}, s_{3}\right)^{T}$. A vector is sometimes regarded as a column matrix. So vector operation such as cross product $(\times)$ and matrix operations such as matrix multiplication are applied to three-dimensional vectors. Matrix operations precede vector operations. 0 denotes a zero vector. For a matrix $A=\left[a_{i j}\right],\|A\|$ denotes the Euclidean norm of the matrix, i.e., $\left\|\left[a_{i j}\right]\right\|=\sqrt{\sum_{i j} a_{i j}^{2}}$. We define a mapping $[\cdot]_{\times}$from a three-dimensional vector to a 3 by 3 matrix:

$$
\left[\left(x_{1}, x_{2}, x_{3}\right)^{T}\right]_{\times}=\left[\begin{array}{ccc}
0 & -x_{3} & 0 \\
x_{3} & 0 & -x_{1} \\
-x_{2} & x_{1} & 0
\end{array}\right] .
$$

Using this mapping, we can express cross operation of two vectors by the matrix multiplication of a 3 by 3 matrix and a column matrix:

$$
X \times Y=[X]_{\times} Y .
$$

The reference system $s$, in which the coordinates of the vector $x$ are expressed, is reported as superscribe on the upper-left corner of $x$ with the notation $x^{s}$.

The reference systems considered in the paper are the following:

i inertial system;

e the ECEF (Earth Cenetered Earth Fixed) system;

n the NED (North East Down) system, tangent to the earth ellipsoid, at a reference Latitude and Longitude $\left(\right.$ Lat $_{0}$, Lat $\left._{0}\right)$;

b the "body" system as seen by the IMU (Inertial Measurement Unit);

c the camera system, with fixed orientation with respect the IMU.

The default coordinate system in which the UAV position, i.e. (Lat, Lon, Alt) of the vehicle center of mass, is given is the ECEF system. $C_{n}^{e}$ is the reference change matrix from NED to ECEF. The attitude and heading of the vehicle is given as $C_{b}^{n}(\phi, \theta, \psi)$ where $C_{b}^{n}$ is the reference change matrix associated with the roll $(\phi)$, pitch $(\theta)$, and yaw $(\psi)$ angles. In general, the camera standard reference system might be rotated with respect to the body reference system. Thus, the transformation from the camera reference to ECEF is given by

$$
C_{c}^{e}=C_{n}^{e}(\text { Lat }, \text { Lon }) C_{b}^{n}(\phi, \theta, \psi) C_{c}^{b}
$$

where $C_{c}^{b}$ represents the constant reference change matrix from camera system $c$ to body unit system $b$.

Let us consider a 2-D plane $\Pi$ in 3-D space. Consider two images taken by the camera at different time. The equation linking two image points $p^{\prime}$ and $p$ which are the projection of the same point of $\Pi$ is:

$$
p^{\prime} \simeq H_{\Pi} p,
$$

where $p^{\prime}=\left[u^{\prime}, v^{\prime}, 1\right]^{T}$ and $p=[u, v, 1]^{T}$ are the (normalized) homogeneous coordinates in the second and the first camera reference frame respectively and the symbol $\simeq$ means that equality is up to a scale factor. We call the matrix

$$
H_{\Pi}=\left(C_{c_{1}}^{c_{2}}+\frac{r_{21}^{c_{2}} N^{T}}{d}\right)
$$

the planar homography matrix induced by the plane $\Pi$. It contains the information about the camera movement $\left(\left[C_{c_{1}}^{c_{2}}, r_{21}^{c_{2}}\right]\right)$ ant the scene structure (the normal to the plane $N$, and the distance $d$ ).

We suppose that the scene structure is known, supposing $N^{n}=$ $(0,0,-1)$ and using an altimeter to determine the distance $d$ of the aerial platform from the ground. Here $\left[C_{c_{1}}^{c_{2}}, r_{21}^{c_{2}}\right]$ represents the rigid transformation (rotation and translation) which brings points ( $i$. $e$. transforms coordinates) from the camera 1 to the camera 2 standard reference system.

The coordinates change equation is given by

$$
x^{c_{2}}=C_{c_{1}}^{c_{2}} x^{c_{1}}+r_{21}^{c_{2}}
$$

where $x^{c_{k}}$ represent the coordinates in the camera $k$ standard reference system.

The translation vector between the two camera center $r_{12}^{s}=O_{2}^{s}-$ $O_{1}^{s}$ expressed in a generic reference system $s$ is equal to $-C_{2}^{s} r_{21}^{c_{2}}$.

The matrix $C_{c_{1}}^{c_{2}}$ contains in it different contributions:

1. the rotation of the camera by the pan-tilt unit;

2. changes in the vehicle attitude and heading;

3. rotation between the two NED systems (in $t_{2}$ and $t_{1}$ ).

Indeed it can be also expressed as

$$
C_{c_{1}}^{c_{2}}=C_{e}^{c_{2}} C_{c_{1}}^{e}=C_{c_{2}}^{e T} C_{c_{1}}^{e}
$$

By means of eq. (3) and with a little algebra we obtain 


$$
C_{c_{1}}^{c_{2}}=C_{b 2}^{c_{2}} C_{n_{2}}^{b_{2}} \underbrace{C_{e}^{n_{2}} C_{n_{1}}^{e}}_{\approx I} C_{b_{1}}^{n_{1}} C_{c_{1}}^{b_{1}}
$$

In the above equation we supposed (Lat,Lon) did not change a lot between two consecutive frames, hence the identity approximation. This also means that the two NED reference systems (i.e., corresponding to $n_{1}$ and $n_{2}$ ) can be thought to be coincident up to a translation. The eq.(8) then reads as

$$
C_{c_{1}}^{c_{2}} \approx C_{b_{2}}^{c_{2}} C_{n_{2}}^{b_{2}} C_{b_{1}}^{n_{1}} C_{c_{1}}^{b_{1}}
$$

Therefore, supposing a fixed camera orientation with respect to the body, the functional dependency is only in the rotation of the body, i.e. in the change of the vehicle attitude and heading,

$$
C_{b_{1}}^{b_{2}} \approx C_{n_{2}}^{b_{2}} C_{b_{1}}^{n_{1}} \approx R(d \phi, d \theta, d \psi)=R_{x}(d \phi) R_{y}(d \theta) R_{z}(d \psi)
$$

where $d \phi=\phi_{2}-\phi_{1}, \quad d \theta=\theta_{2}-\theta_{1}$ and $d \psi=\psi_{2}-\psi_{1}$.

The notation $R_{a}(\alpha)$ denotes the matrix describing the rotation along $a$ axis of angle $\alpha$.

\section{X264 MODIFICATIONS FOR MOTION DATA EXTRACTION}

This section presents the changes made to the $\mathrm{x} 264$ encoder (core 142) in order to allow the extraction of the motion data computed during the compression procedure. The modifications do not alter the result video bitstream and the output is still an H.264/AVC compliant stream. A brief preliminary discussion on the x264 library (VideoLan, 2014) is reported followed by the proposed modifications.

The encoder consists of three main stages: the lookahead block, which is responsible, on the basis of user-selected parameters and temporally neighboring frames, to decide each frames type (Intra, Predicted or Bi-predicted) and the proper quantization step; the analysis block, which analyses each macroblock (MB) within the frame to select the more convenient prediction type (Inter or Intra) and computes all the information required for motion compensation; the coding block, which writes in the output bitstream the information related to each MB. All the motion information related to the motion compensation of each MB (such as partitions, MVs and reference frames) is available into the x264_macroblock_analyse function. This function performs an analysis according to the frame type. In the case of I frame, only spatial prediction modes are tested, checking which neighbours are available and computing for each mode and for each possible partition a cost. Then, the combination of partition and prediction that maximize the quality and minimize the coding rate is selected. In the case of $\mathrm{P}$ and $\mathrm{B}$ frames the procedure is similar, but Inter prediction is also tested, computing a MV for each available reference frame. Motion compensation is initially applied to $16 \times 16 \mathrm{MBs}$ and then for each possible partition. The partitions with the minimum cost are selected, each one with its correspondent MV and reference. All these motion data are then copied in a cache structure within the mb structure, which contains all the information related to the current $\mathrm{MB}$, and the following $\mathrm{x} 264$ macroblock_encode function write them into the bitstream. Within the cache structure, the reference indexes and the MVs are stored in two different integer arrays, i.e. ref and mv. The bi-dimensional ref array contains the frame reference indexes. The first dimension of the array refers to the reference list, namely L0 for previous reference frames (used for both $\mathrm{P}$ and $\mathrm{B}$ frames) and $\mathrm{L} 1$ for future reference frames (used only by $\mathrm{B}$ frames). The second dimension refers to the spatial position of the partition within the MB. Since the minimum partition in H.264/AVC standard is $4 \times 4$ then there are 16 different indexes. If a bigger partition is selected by the encoder for that specific $\mathrm{MB}$, all the reference indexes are duplicated. The array mv contains the MV of each MB. The first and the second dimension are as before, while the third dimension distinguishes the horizontal and vertical component of the MV. Once the encoder has finished encoding a MB, it starts with the following one and all the motion data stored in the mb structure is overwritten. The proposed framework implements at this stage a function whose task is to save all the motion information related to every MB. The preserved parameters are MVs, reference indexes, MB type, partitions and position within the frame.

\section{METADATA CORRECTION BY VISION AIDED SENSOR FUSION}

The overall data fusion architecture is sketched in Figure 4, where the sensor fusion block implements the Kalman filtering of the data provided by the Navigation System and the camera egomotion data from the video processing system. The camera egomotion module is based on the homography matrix, which relates homologous points in two different views of the same scene. In this work the correspondences are given by the refined motion vector provided by the encoder.

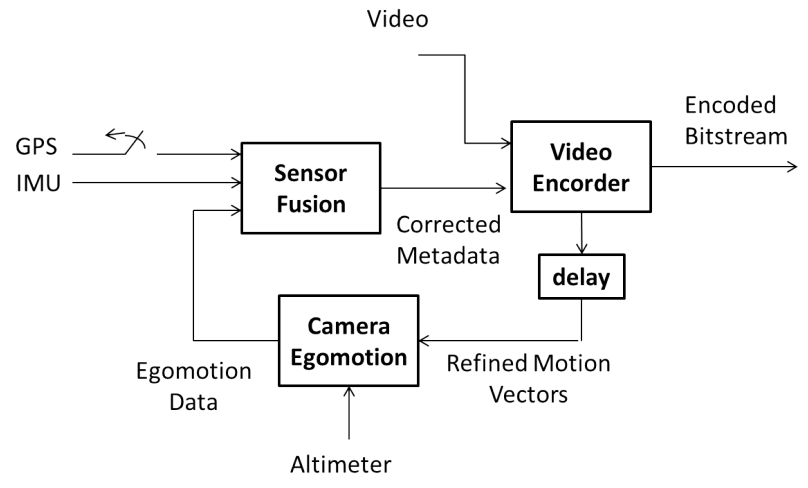

Figure 4: Metadata improvement by sensor fusion.

From the motion vectors calculated by the encoder, after the motion refinement step, the homography matrix is estimated and then decomposed into his motion and structure parameters. The homography estimation (based on the RANSAC algorithm) and decomposition procedure is behind the scope of this work and will be omitted. The interested reader may refer to (Hartley and Zisserman, 2004) and the reference therein.

The final purpose of the proposed sensor fusion algorithm is the estimation of the position and attitude of the camera, supposed internally and externally calibrated. The data fusion algorithm is based on the (UKF) (Van Der Merwe and Wan, 2003) (Julier and Uhlmann, 2004), because dynamic and observation equations are non-linear in their original form. Like all the Kalman Filters, an UKF consists of two steps: model forecast and data assimilation. Sigma points are used to represent the current state distribution and to propagate the distribution to the next state and to the output. Mean and covariance of the transformed sigma points can 
be used to calculate the Kalman gain and to update the state prediction. Often such a filter has been used to estimate the pose of an UAV. In particular, in this work, we adopt the same solution proposed in (Angelino et al., 2013a).

Angular velocities and linear accelerations provided by the IMU are used in the Kalman prediction step. GPS position and speed as well as camera egomotion parameters are used in the Kalman update step, in order to correct the position and the orientation drift due to the integration of the IMU data. With respect to (Angelino et al., 2013a) a magnetometer is added, in order to correct the heading.

The state vector includes the position and speed of the aircraft UAV (eq. 11 and 13) in the tangent reference frame, and the rotation matrix from body to tangent reference frame (eq. 14). Moreover, the state vector includes also two variables relative to the delayed aircraft position and the delayed rotation matrix (eq. 12 and 15). The delay is related to the video coding frequency $f_{E N C}$. The UKF is applied to estimate the state variables. The input measurements for prediction of state variables are the inertial accelerations $\tilde{a}^{b}$ and the angular speeds $\tilde{\Omega}^{b}$ processed by the IMU. Therefore, we have:

$$
\left\{\begin{array}{l}
x^{n}(k+1)=x^{n}(k)+v^{n}(k) \Delta T_{i} \\
\bar{x}^{n}(k+1)=x^{n}(k-\Delta k) \\
v^{n}(k+1)=v^{n}(k)+C_{b}^{n}(k) \tilde{a}^{b}(k) \Delta T_{i} \\
C_{b}^{n}(k+1)=C_{b}^{n}(k)\left[I+\tilde{\Omega}^{b}(k) \Delta T_{i}\right] \\
\bar{C}_{b}^{n}(k+1)=C_{b}^{n}(k-\Delta k)
\end{array}\right.
$$

Output equations are based on the following measurements:

- GPS antenna position $x_{a}^{e}$ in the ECEF reference frame (eq. $16)$;

- GPS speed $v_{a}^{n}$ in the NED reference frame (eq. 17);

- Magnetic heading $\psi_{\mu}$ (eq. 18);

- Camera center position change $\Delta \bar{x}^{n}$ in the tangent reference frame (eq. 19);

- Camera attitude change $\Delta \bar{C}^{b}$ respect to the tangent reference frame (eq. 20-22).

$$
\left\{\begin{aligned}
x_{a}^{e}(k) & =C_{n}^{e}(k)\left[x^{n}(k)+C_{b}^{n} \Delta x_{a}^{b}\right]+x_{a}^{e}\left(k_{0}\right) \\
v_{a}^{n}(k) & =v^{n}(k)+C_{b}^{n}(k) \tilde{\Omega}^{b}(k) \Delta x_{a}^{b} \\
\psi_{\mu}(k) & =\psi(k) \\
\Delta \bar{x}^{n}(k) & =\left[x^{n}(k)-\bar{x}^{n}(k)\right] \\
\left.\Delta \bar{C}^{b}(k)\right)\left.\right|_{(1,2)} & =\left[C_{b}^{n}(k)^{-1} \bar{C}_{b}^{n}(k)\right]_{(1,2)} \\
\left.\Delta \bar{C}^{b}(k)\right|_{(1,3)} & =\left[C_{b}^{n}(k)^{-1} \bar{C}_{b}^{n}(k)\right]_{(1,3)} \\
\left.\Delta \bar{C}^{b}(k)\right)\left.\right|_{(2,3)} & =\left[C_{b}^{n}(k)^{-1} \bar{C}_{b}^{n}(k)\right]_{(2,3)}
\end{aligned}\right.
$$

The state vector equations (11-15) update at the $f_{I M U}$ rate, while equations (16-18) and (19-22) update respectively at $f_{G P S}$ and $f_{E N C}$ rates. When Intra coding is performed, the Kalman update step based on camera egomotion (eq. 19-22) is skipped.

\section{EXPERIMENTAL RESULTS}

\subsection{Test video sequences}

Three different aerial sequences (ISMB/CIRA, 2013) have been encoded and then their motion data have been processed. We considered low frame rate sequences $(0.5-1 \mathrm{fps})$ and relative long MVs as this is often the case for UAV acquired high resolution video sequences. The characteristics of the three sequences are reported in Table 1.

\begin{tabular}{|l|c|c|c|c|c|}
\hline Video Seq. & FR & Res & h-FOV & Speed & Alt \\
\hline & {$[\mathbf{f p s}]$} & {$[\mathbf{p i x} \times \mathbf{~ p i x}]$} & {$[\mathbf{d e g}]$} & {$[\mathbf{k m} / \mathbf{h}]$} & {$[\mathbf{m}]$} \\
\hline Camp Pend. & 1 & $1088 \times 672$ & 60 & 250 & 800 \\
\hline Rome & 0.5 & $1088 \times 672$ & 60 & 250 & 800 \\
\hline Brezza & 1 & $3000 \times 2000$ & 73.7 & 2 & 80 \\
\hline
\end{tabular}

Table 1: Aerial video sequences characteristics.

The sequences "Camp Pendleton" (a snapshot in Figure 5) and "Rome" (a snapshot in Figure 6) have been generated using Google Earth. In the "Camp Pendleton" sequence, the overflight region is a military base and the surrounding areas. The area is substantially homogeneous and with few details. The "Rome" sequence refers to a flight over the city of Rome, rich of details. For the simulated sequences the horizontal Field Of View is 60 degrees, the frame resolution is $1088 \times 672$ pixels. The flying altitude is $800 \mathrm{~m}$ for both the simulated video sequences. The "Brezza" 7 sequence is part of a video recorded using a real multi-rotorcraft mini-UAV over a rural region poor of details (grass with some trees and only a few of man-made structures). The frame resolution is $3000 \times 2000$ pixels. The flying altitude $(80 \mathrm{~m})$ is much lower than the simulated video sequences. The horizontal FOV is 73.7 degrees.

Ground truth metadata are provided by the image generator for the synthetic video sequences, while for the "Brezza" sequence, they are estimated from multiple views by a bundle adjustment technique. However, in the experiments a noisy version of these metadata has been generated according to the sensors model described in (Angelino et al., 2013a). The parameters of the sensors model are extracted by the datasheet of a well known commercial GPS aided Attitude and Heading Reference System commonly employed in aeronautical applications.

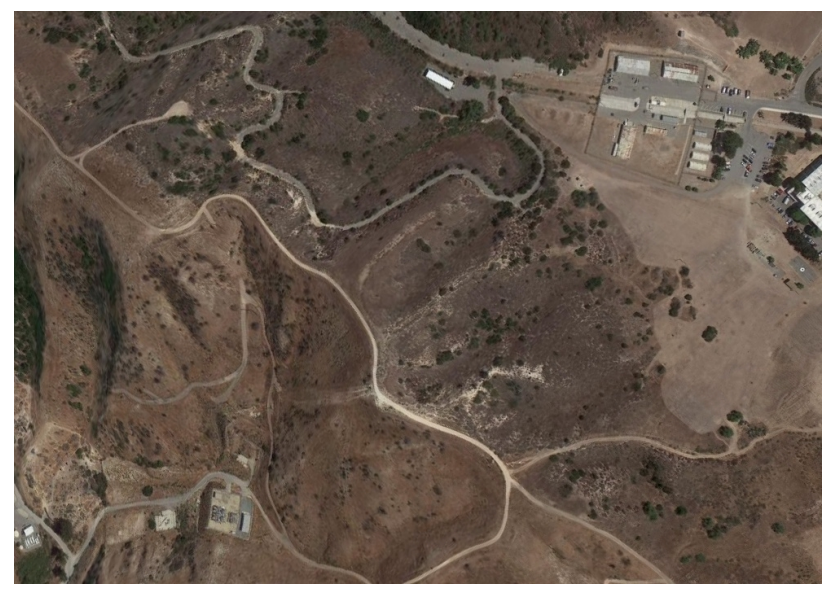

Figure 5: A snapshot from the sequence Camp Pendleton, as simulated in Google Earth, with a 1088x672 resolution.

\subsection{Data fusion performance}

The performance of the proposed data correction approach has been previously discussed in (Angelino et al., 2013a). Here we 


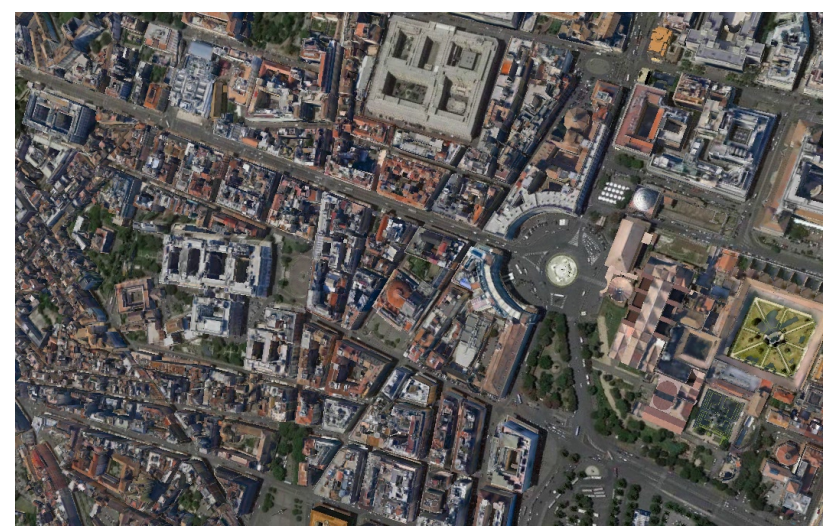

Figure 6: A snapshot from the sequence Roma, as simulated in Google Earth, with a 1088x672 resolution.

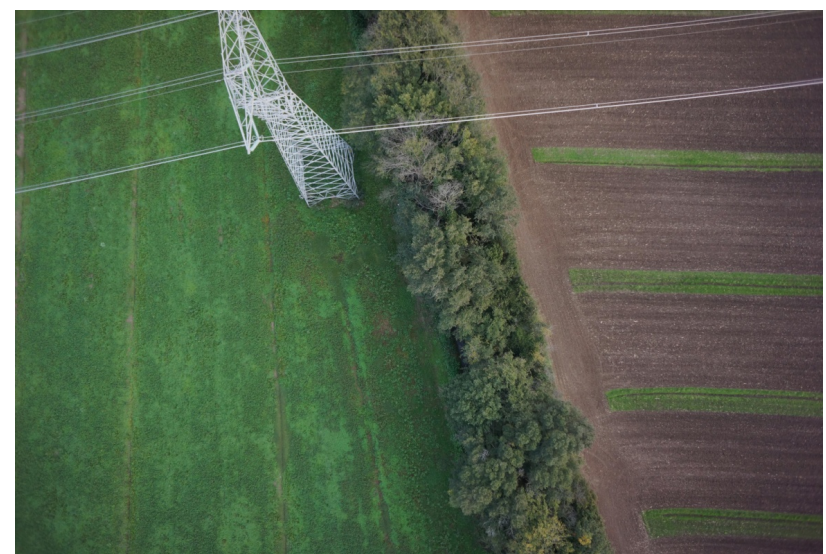

Figure 7: A snapshot from the sequence Brezza, with a $3000 \times 2000$ resolution.

report the main conclusions on the obtained results. The working conditions of the experiments showed in the Figures 8-10 (i.e. sensors performance) are the same as reported in (Angelino et al., 2013a). The test video sequence is Rome. In the reported experiments, the IMU sampling rate is supposed $100 \mathrm{~Hz}$, the GPS data update is $10 \mathrm{~Hz}$, the camera egomotion processing rate is 5 $\mathrm{Hz}$.

The black line represents the ground truth while the red line represents the estimation performed with the Kalman Filter. In order to outline the IMU drift phenomena, the direct integration of the IMU data (Kalman prediction equations only) is represented with a blue line. In this case the corrections performed with the measures coming from GPS, magnetometer and camera (Kalman update equations), are completely skipped.

Figure 8 shows the UAV attitude estimation in terms of pitch angle using the UKF. Estimation of Euler angles is independent by GPS measurements. This is due to the choice of installing the GPS antenna at aircraft c.g., in order to highlight the advantages in the application of camera measurements (GPS measurements can be used to estimate attitude only in the presence of a sufficient lever arm). In this situation GPS measurements do not provide observability of attitude error.

Figures 9 and 10 show the UAV position and speed estimation along the North direction. As in the attitude estimation, speeds and positions are also estimated with very good performances. The simulation results show that the position and orientation data can be recovered with high precision with such sensor fusion strategy.

Other experiments have concerned use of the output camera equation alone, in order to simulate GPS outages. The curves are reported in magenta in the same Figures 8-10. Attitude estimation, as already explained, is not influenced by GPS. GPS speed, and hence the position, is estimated with degraded performances. However, estimation allows the flight continuation for some time with a reasonable position error. The same sensors model has been used in the experiments concerning video coding, reported in Subsection 5.4 .

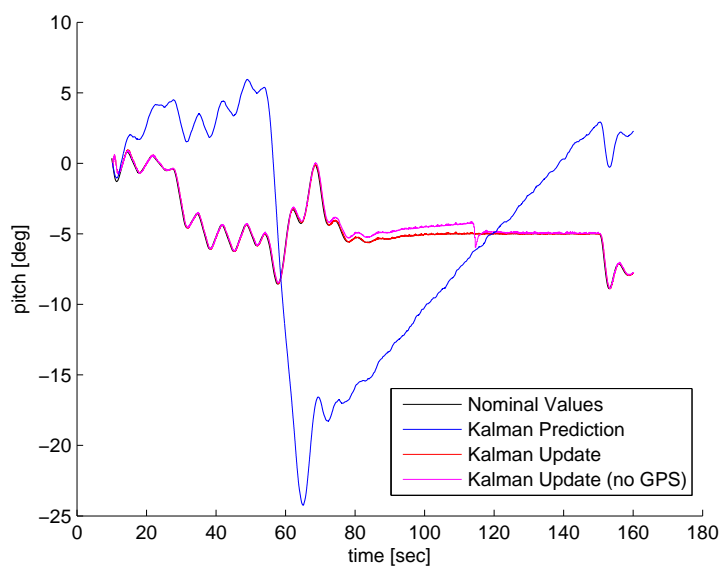

Figure 8: Pitch angle estimation (Roma Sequence). Nominal value (black line), Kalman Prediction without GPS (blue line), Kalman Update (red line), Kalman Update without GPS (magenta line).

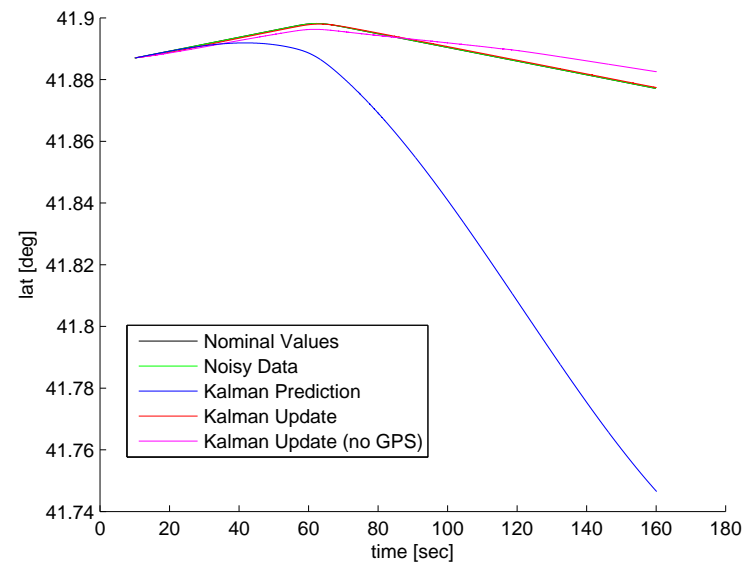

Figure 9: Latitude estimation (Roma Sequence). Nominal value (black line), Kalman Prediction without GPS (blue line), Kalman Update (red line), Kalman Update without GPS (magenta line).

\subsection{Encoder settings}

The x264 library offers several presets. Each preset is a collection of parameters which are set in order to get a good trade-off between quality and coding time for different application scenarios. The "medium" preset is general purpose, and is compatible with low computationally demanding scenarios. Because in this scenario the video frame rate is very low (i.e. $0.5 \mathrm{fps}$ ), a time demanding preset, i.e. the "slower", can be also considered, in 


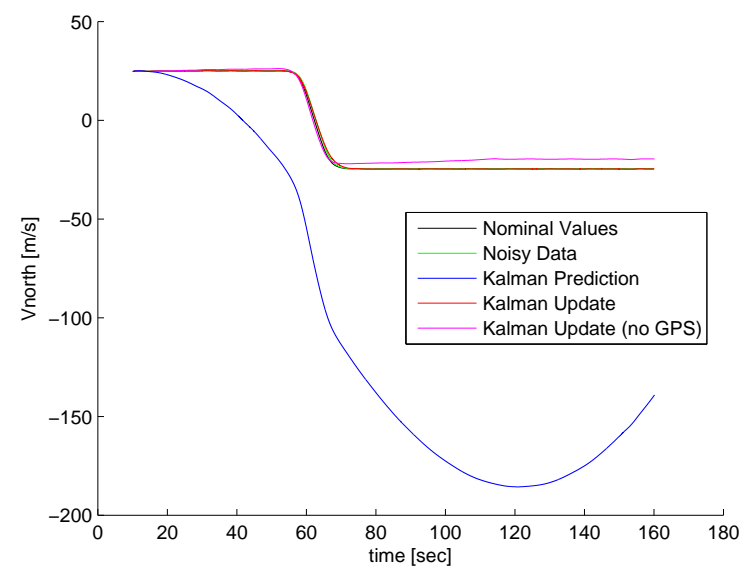

Figure 10: Linear speed in North direction (Roma Sequence). Nominal value (black line), Kalman Prediction without GPS (blue line), Kalman Update (red line), Kalman Update without GPS (magenta line).

order to reach better encoding quality. These preset options for the proposed modified x 264 encoder are labeled in the figures as "medium" and "slower", instead the same configurations in the original x264 encoder are labeled with the prefix "x264", and are respectively "x264_medium" and "x264_slower".

The sensor aided encoder often uses only one reference frame in the GOP, because the low overlap among the frames. For this reason, the comparison with the reference $\mathrm{x} 264$ encoder with only one reference frame in the GOP is presented. In this case only the "medium" preset is reported. The corresponding label is "x264_medium_ref1".

Two other coding option are specifically presented for the proposed sensor aided encoder. A first option excludes the refinement step of the motion estimation trough video analysis. This option is labeled as "medium_nors", where the word "medium" indicates the used preset and the acronym "nors" is for NO Refinement Search.

A further option is added in order to force the sensor aided encoder to perform the motion estimation also when Intra coding is possible. This processing step can be useful in order to produce more accurate motion fields that can be used by the sensor fusion module. These experiments, reported for the preset "medium", are labeled as "medium_uem" or "medium_nors_uem", where the latter acronym is for Use Estimated Motion vectors.

\subsection{R-D performance improvements}

In this section the Rate-Distortion (R-D) performance of the proposed sensor aided encoder, using corrected metadata, is compared to that of the x264 implementation of h.264. Eight ratedistortion curves are plotted in the Figures 11-13 for each test video sequence. On the x-label the encoding bitrate is reported, while on the y-label the PSNR (Peak Signal to Noise Ratio), that is a commonly used objective video quality measure.

A first observation is that the proposed sensor aided encoder outperforms the reference $\mathrm{x} 264$ encoder both with the medium and the slower preset. For example, for the "Camp Pendleton" video sequence, at $400 \mathrm{kbps}$, the PSNR of the proposed encoder is 35.41 $\mathrm{dB}$ versus $34.76 \mathrm{~dB}$ of the reference with the medium preset, and $36.24 \mathrm{~dB}$ versus $34.31 \mathrm{~dB}$, with the slower preset. For the sequence "Rome", at the same bitrate of $400 \mathrm{kbps}$, the PSNR of the sensor aided encoder is $33.93 \mathrm{~dB}$ versus $32.74 \mathrm{~dB}$ of the reference with the medium preset, and is $35.18 \mathrm{~dB}$ versus $32.50 \mathrm{~dB}$ for the slower preset.

The proposed sensor aided encoder has a similar behaviour on real video sequences also. On the sequence "Brezza", for example, at $3250 \mathrm{kbps}$, the PSNR is $35.12 \mathrm{~dB}$ versus $34.48 \mathrm{~dB}$ of $\mathrm{x} 264$.

It is worth to notice that the reference $\mathrm{x} 264$ encoder uses a complex GOP analysis in order to optimize the use of the I, P and B frames. The proposed sensor aided implementation instead, at the current stage of development, uses a more simple strategy based on only a I frame per GOP and all P frames (this strategy is reasonable, due to the continuity of the camera motion). For this reason it is more correct to compare the "medium" curve with the "x264_medium_1Ref" curve, instead of the "x264_medium" curve. Comparing these couples of curves, the proposed solution can be further appreciated.

From the figures it is also possible to note that the "slower" preset has lower quality than "medium" preset for the reference x264 encoder. This is due to the large number of B-frames selected by the $\mathrm{x} 264$ GOP decision algorithm. In the considered scenarios, in which there is low overlap among successive frames, the use of B frames has bad effects on the output quality. The proposed sensor aided implementation, instead, uses the same GOP structure for the two different presets, that is similar to the best option selected by $\mathrm{x} 264$.

Further considerations can be made by analyzing only the the sensor aided implementations. Indeed, by comparing the "medium" and "medium_nors" curves, it is clear that the motion search refinement step, based on video analysis, is essential to reach high rate distortion performance. On the contrary, by comparing the "medium_nors" and "medium_nors_uem" curves, it is possible to conclude that a pure motion estimation approach cannot be preferred to a combined strategy, based both on Intra and Inter block prediction, at least in the case in which video analysis is not used to refine the motion vectors. The comparison between the "medium" and the "medium_uem" curves, however show that, using the video analysis for motion vectors refinement, also don't considering the Intra option, it is possible to reach a performance near to the combined approach (both Intra and Inter blocks). In the case of Camp Pendleton and Rome, the gap is negligible.

As final remark, we would like to point out that the metadata correction performance is generally very good. Indeed, the standard deviation of the orientation estimation error results less than 0.2 degrees for all video sequences. As a consequence, the encoding results are very similar to the ones obtained using the ground truth (difference in PSNR lesser than $0.15 \mathrm{~dB}$ ). For this reason, a comparison of R-D curves between ground truth and corrected metadata is not shown.

\section{CONCLUSIONS}

In this work we proposed an integrated solution of sensor aided video encoder, able to process corrected metadata in order to estimate the global motion in aerial video sequences, strongly reducing the need of video analysis. A novel encoder architecture is presented and a fully h.264 implementation is proposed and tested, on simulated and real video sequences. The experimental results show the effectiveness of the proposed solution at high resolution and low frame rates. The suggested applications are to UAV imagery transmission and storage, under channel capacity or power supply constraints. Future works will be focused on computational complexity aspects and on optimized solutions for high speed vision based metadata corrections. 


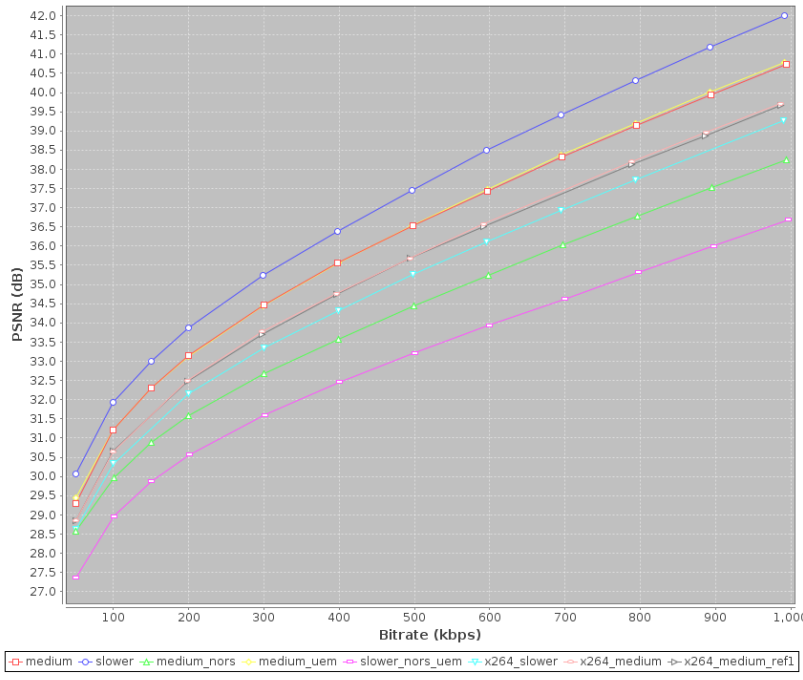

Figure 11: R-D Curves for the "Camp Pendleton" sequence, with a resolution of $1088 \times 672$ pixels and a frame rate of $1 \mathrm{fps}$.

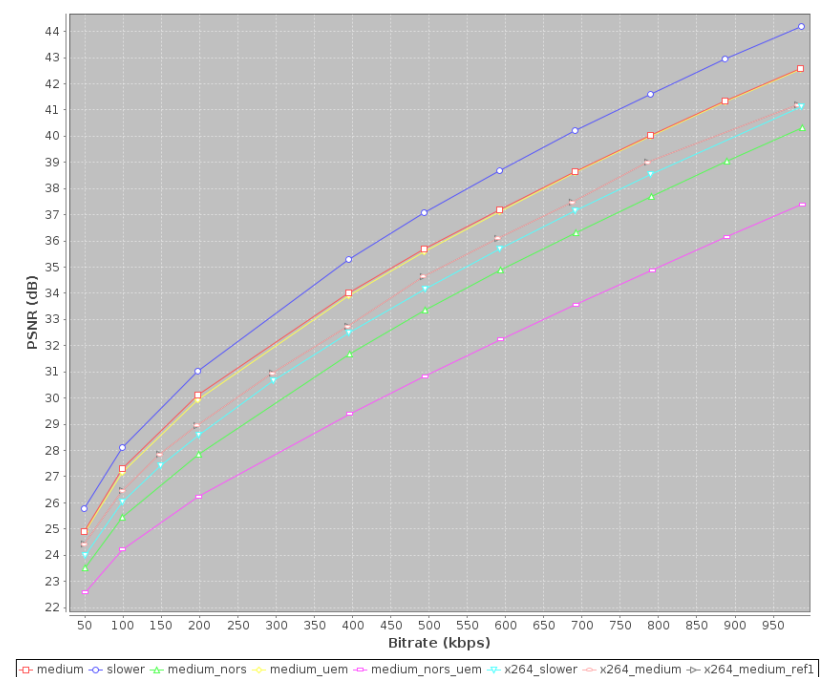

Figure 12: R-D curves for the "Rome" sequence, with a resolution of $1088 \times 672$ pixels and a frame rate of $0.5 \mathrm{fps}$.

\section{REFERENCES}

Angelino, C. V., Baraniello, V. R. and Cicala, L., 2012. Uav position and attitude estimation using imu, gnss and camera. In: Proceeding of the 15th International Conference on Information Fusion (FUSION), Singapore, Singapore, pp. 735-742.

Angelino, C. V., Baraniello, V. R. and Cicala, L., 2013a. High altitude uav navigation using imu, gps and camera. In: Proceedings of the 16th International Conference onInformation Fusion (FUSION), Istanbul, Turkey, pp. 647-654.

Angelino, C. V., Cicala, L., De Mizio, M., Leoncini, P., Baccaglini, E., Gavelli, M., Raimondo, N. and Scopigno, R., 2013b. Sensor aided h.264 video encoder for uav applications. In: Proceedings of the 30th Picture Coding Symposium (PCS), pp. 173-176.

Bhaskaranand, M. and Gibson, J., 2015. Global motion assisted low complexity video encoding for uav applications. IEEE Journal of Selected Topics in Signal Processing 9(1), pp. 139-150.

Chen, X.-1., Zhang, S.-c. and Liu, J., 2011. Design of uav video compression system based on h.264 encoding algorithm. In: Proceedings of the 1st International Conference on Electronic and

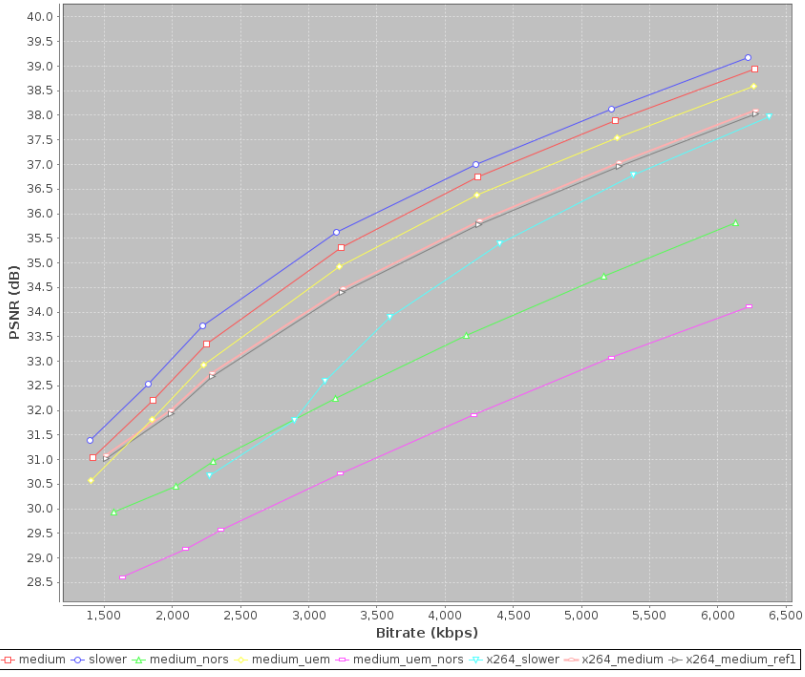

Figure 13: R-D curves for the "Brezza" sequence, with a resolution of 3000x2000 pixels and a frame rate of $1 \mathrm{fps}$.

Mechanical Engineering and Information Technology (EMEIT), Vol. 5, Harbin, China, pp. 2619-2622.

Gong, J., Zheng, C., Tian, J. and Wu, D., 2010. An imagesequence compressing algorithm based on homography transformation for unmanned aerial vehicle. In: Proceedings of the 1st International Symposium on Intelligence Information Processing and Trusted Computing (IPTC), Huanggang, China, pp. 37-40.

Hartley, R. I. and Zisserman, A., 2004. Multiple View Geometry in Computer Vision. Second edn, Cambridge University Press, ISBN: 0521540518.

ISMB/CIRA, 2013. Test sequences [online] available at: http://www.ismb.it/mise_cira.

ISO/IEC, 2006. ISO/IEC International Standard 14496-10, Information Technology Coding of Audio-Visual Objects Part 10: Advanced Video Coding. Third edn, ISO/IEC.

Julier, S. J. and Uhlmann, J. K., 2004. Unscented filtering and nonlinear estimation. Proceedings of the IEEE 92(3), pp. 401422.

Merritt, L. and Rahul, V., 2006. X264: A high performance h.264/avc encoder [online] available at:

http://neuron2.net/library/avc/overview_x264_v8_5.pdf.

Morimoto, C., Burlina, P. and Chellappa, R., 1997. Video coding using hybrid motion compensation. In: Proceedings of the 4th International Conference on Image Processing (ICIP), Vol. 1, Santa Barbara, California, USA, pp. 89-92.

NATO, 2009. Stanag 4609, nato digital motion imagery format [online] available at http://www.nato.int/structur/ac/224/standard/4609/4609documents/4609eed03.pdf.

Soares, P. H. F. T. and Pinho, M. d. S., 2013. Video compression for uav applications using a global motion estimation in the h.264 standard. In: Proceedings of the 6th International Workshop on Telecommunications, Vol. 1, Santa Rita do Sapucaì, Brazil.

Steinbach, E., Wiegand, T. and Girod, B., 1999. Using multiple global motion models for improved block-based video coding. In: Proceedings of the 6th International Conference on Image Processing (ICIP), Vol. 2, Kobe, Japan, pp. 56-60.

Van Der Merwe, R. and Wan, E., 2003. Sigma-point kalman filters for probabilistic inference in dynamic state-space models. In: Proceedings of the Workshop on Advances in Machine Learning, Montreal, Canada.

VideoLan, 2014. x264 software, core 142 [online] available at: http://www.videolan.org/developers/x264.htm. 